\title{
Welche konservativen Behandlungsverfahren sind heute noch gültig?
}

\author{
J. Blum, A. Noltze, P. M. Rommens
}

\section{Zusammenfassung}

Neue, effektivere und schonendere operative Verfahren haben in der Unfallchirurgie eine Reihe konservativer Verfahren verdrängt. Dennoch ist der Stellenwert der konservativen Behandlung auch heute unvermindert stabil und im Repertoire eines Unfallchirurgen unverzichtbar. Eine Reihe subtiler und sehr effektiver Gipstechniken scheint durch die primär operative Tätigkeit vieler Unfallchirurgen in Gefahr, an Qualität und Effizienz mangels Training zu verlieren. Wichtig ist, die Überlegenheit des einzelnen Verfahrens für die jeweils spezifische Situation herauszuarbeiten. Dieser Prozess fußt auf unfallchirurgischer Erfahrung, kritischem Abwägen in Anpassung an die jeweilige Frakturund Weichteilsituation, aber auch an die Gesamtsituation des Patienten einschließlich dessen Persönlichkeit. Reposition, Retention und Rehabilitation müssen bei Wahl des konservativen Behandlungsweges erkennbar zur knöchernen Ausheilung in möglichst anatomischer Stellung führen können und eine zügige Mobilisation gewährleisten. Dies bringt die konservativen Konzepte vor allem bei stabilen und undislozierten Frakturen zur Anwendung. Die oberen Extremitäten bieten sich deutlicher als die unteren an. Komplikationen der konservativen Behandlung, wie Drucknekrosen, vaskuläre Beeinträchtigungen, Thrombosen, Embolien, ischämische Muskelkontrakturen und Pseudarthrosen dürfen nicht unterschätzt werden und sollten vermieden bzw. früh erkannt und behandelt werden.

\section{Einleitung}

Ein Beitrag über den aktuellen Stand der konservativen Knochenbruchbehandlung in einem OP-Journal stellt nur einen scheinbaren Widerspruch dar. Es gibt viele gute Gründe, sich auch als primär operativ tätiger Arzt/Ärztin wie auch OP-Pfleger/in mit den heute gültigen Richtlinien konservativer Behandlungsverfahren auseinander zu setzen.

Der rasante Aufschwung der operativen Knochenbruchbehandlung vom Ende des 19. Jahrhunderts bis heute - und hier insbesondere die Entwicklung nach dem Zweiten Weltkrieg - mag das Augenmerk der Fachwelt wie auch der unfallchirurgischen Laien vorzugsweise auf

OP-JOURNAL 2000; 16: 130-136

(c) Georg Thieme Verlag Stuttgart · New York operative Verfahren lenken, insbesondere auf die spektakulärsten Errungenschaften der modernen Osteosynthesetechniken und ihre Implantatsysteme. Die archaischen Wurzeln verhafteten konservativen Verfahren mögen oberflächlich betrachtet anachronistisch wirken. Manche Kritiker stellen deren Indikationsstellung teilweise in Verbindung mit mangelndem operativen Vermögen. Dies führt bis zum heutigen Zeitpunkt zu einer Entwicklung in manchen unfallchirurgischen Kliniken und Praxen, in der die subtilen Konzepte und Fertigkeiten konservativer Bruchbehandlung durch Unterschätzung an Qualität und Hingabe leiden und im Sinne der „self-fullfilling prophecy“ dann auch mit schlechten Ergebnissen behaftet sein können.

Tatsächlich ist aber der Stellenwert konservativer Knochenbruchbehandlung auch gerade heute nicht hoch genug einzuschätzen. Selbstverständlich unterliegt die Entscheidung zu einem solchen Verfahren der gleichen kritischen Überlegung und Einschätzung, wie zu einem bestimmten operativen Konzept. Zusätzlich ist in Zeiten schrumpfender finanzieller Ressourcen im Einzelfall abzuwägen, ob ein teueres operatives Verfahren in der Tat erkennbare Vorteile gegenüber einem ökonomisch günstigeren konservativen Behandlungskonzept bietet. Genauso sind biologische und persönlichkeitsbezogene Gesichtspunkte des Patienten einzubringen. Hinzu kommt, dass generell bei Frakturen im Wachstumsalter, wo möglich und sinnvoll, den konservativen Verfahren der Vorzug gegeben wird.

Für Instrumentierpfleger/innnen wie auch Springer im OP ist es gleichermaßen bedeutsam, die Grundkonzepte der konservativen Verfahren zu kennen. Dies erleichtert den Dialog mit dem Chirurgen und auch das Verständnis für die Wahl bestimmter operativer Konzepte. Darüber hinaus findet sich auch im OP eine Reihe von Überschneidungen mit konservativen Behandlungstechniken - bei einigen operativen Verfahren wird bereits im OP mit ruhigstellenden Verbänden und Gipsen kombiniert, viele Unfallpatienten werden mit temporären Verbänden und Gipsfixationen in den $\mathrm{OP}$ eingeschleust.

\section{Grundkonzepte konservativer Frakturbehandlung}

Die klassischen Trias der konservativen Knochenbruchbehandlung - Reposition, Retention, Rehabilitation - sind nach wie vor gültig.

Unter Reposition wird die Einrichtung des Bruches in jene Stellung verstanden, in der der Bruch ausheilen soll. Die Retention bezeichnet das Festhalten dieses Repositionsergebnisses, bis die Knochenbruchheilung eingetreten ist. Die Rehabilitation beinhaltet die Übungsbehandlung, um die Funktionsbehinderung möglichst klein zu halten. 
Die Reposition hat möglichst früh zu erfolgen. In dieser Frühphase findet man noch eine relativ entspannte Muskulatur. Von wesentlicher Bedeutung ist hierbei die ausreichende Schmerzausschaltung. Unter der Vorgabe, das periphere Fragment auf das zentrale Fragment zu stellen, wird die Beseitigung von Achsenknicken, Drehfehlern und Distraktionen angestrebt. Seitverschiebungen um Kortikalisbreite und geringe Verkürzung können je nach Lokalisation und Situation ggf. toleriert werden.

Unter Umständen genügt die manuelle Reposition nicht, um diese Ziele zu erreichen. Je nach Frakturtyp steht eine Reihe kurz- oder längerfristiger Extensionsverfahren zur Verfügung. Nach heutigen Gesichtspunkten treten allerdings konservative Behandlungskonzepte mit langfristigen Extensionen deutlich in den Hintergrund, was sich aus den verbesserten operativen Behandlungskonzepten mit gesteigertem Patientenkomfort und reduzierten Komplikationsraten aufgrund der geringeren Liegedauer erklärt. Dies gilt im übrigen heute auch tendenziell in der konservativen Behandlung kindlicher Frakturen.

Weichteil- und Knochenfragmentinterpositionen zeigen die Grenzen geschlossener Repositionsmöglichkeiten auf.

Zur Retention steht ein weites Spektrum von Gipsfixationen, wie auch retinierender Verbände und Extensionen zur Verfügung. Letztere haben aus oben genannten Gründen an Bedeutung verloren, was für Weichteilextensionen, wie auch für direkte Knochenextensionen gleichermaßen gilt.

Neben der rein funktionellen konservativen Behandlung, die lediglich in ausgewählten Fällen undislozierter, stabiler Bruchformen ohne ein zusätzliches ruhigstellendes Verfahren auskommt, können insbesondere an der oberen Extremität Rucksackverbände, Dessault-, Gilchrist- und andere Verbände bei richtiger Indikationsstellung eine gipsfreie Behandlung ermöglichen.

Der Gipsverband selbst als „Klassiker“ steht auch heute noch im Mittelpunkt konservativer Bruchbehandlungen, wenn auch die verwendeten Materialien sich stellenweise vom klassischen Gips hin zu Kunststoffen gewandelt haben.

Mit Ausnahme der distalen Radiusfraktur und der Sprunggelenkfraktur werden in der Regel beide benachbarten Gelenke in die Fixation miteinbezogen. Eine weitere Ausnahme stellt der Sarmiento-Cast bei der Behandlung unverschobener Unterschenkelquerfrakturen dar.

Auch moderne Materialien entbinden nicht davon, solche Fixationen zu unterpolstern.

Der damit verbundene Nachteil der Verschiebemöglichkeit zwischen Gips, Weichteilen und Knochen muss bei der Wahl des Verfahrens bedacht werden.

Der Stellenwert der Rehabilitationsbehandlung ist auch heute sowohl in der konservativen als auch operativen Behandlung unumstritten. Dies beinhaltet den möglichst frühzeitigen Beginn aktiver krankengymnastischer Übungen, wie auch die Hochlagerung betroffener Extremitäten, um der Schwellneigung entgegen zu wirken. Durch den Gips inaktivierte Muskelgruppen werden durch isometrische Spannungsübungen trainiert, alle nicht ruhiggestellten Gelenke vom ersten Tag an mobilisiert. Mit der Abnahme des Gipses erweitert sich die Rehabilitationsbehandlung auf die zuvor immobilisierten Gelenke und Muskeln. Es gilt, Gelenkversteifungen, Knochenentkalkungen und Muskelatrophien zu vermeiden.

\section{Konservative Frakturbehandlung an der oberen Extremität}

Aufgrund der im Vergleich zu der unteren Extremität geringeren axialen Belastung durch das Körpergewicht gestaltet sich hier der Spielraum hinsichtlich der konservativen Behandlung als größer. Dennoch wäre es übereilt, die obere Extremität auch heute als eine Domäne der konservativen Behandlung im Falle von Knochenbrüchen zu betrachten. Weiterentwicklungen und Verfeinerungen osteosynthetischer Verfahren haben in den letzten Jahren eine Indikationserweiterung zum operativen Konzept an der oberen Extremität bewirkt.

Der überwiegende Teil der Claviculafrakturen wird auch heute noch konservativ versorgt. So ist in den meisten Fällen ein Rucksackverband für 3-6 Wochen ausreichend, wenn wöchentliche Kontrollen und Nachspannen des Verbandes gewährleistet sind. Die Grenzen der konservativen Behandlung sind erreicht, wenn offene Frakturen, drohende Hautdurchspießung oder Gefäß-Nervenverletzungen begleitend vorliegen. Ebenso können dislozierte sehr lateral oder medial gelegene Frakturen kaum im Rucksackverband retiniert werden.

Für die proximale Humerusfraktur (Abb.1) beschränkt sich die konservative frühfunktionelle Behandlung auf übungsstabile Frakturen, bei denen die Kontur des Oberarmkopfes, einschließlich der Tuberkula keine gröberen Verformungen aufweist, die das Gleiten des Kopfes gegenüber dem Pfanne behindern oder zum Einklemmungssyndrom führen können. Im hohen Alter wird man die Indikation zum konservativen Verfahren etwas weiter stellen.

In der konservativen Behandlung der $\mathrm{Hu}$ merusschaftfrakturen werden teilweise sehr gute Heilungsraten beschrieben (Abb.2). Dies hängt mit der Ummantelung des Humerusschaftes mit gut durchbluteter Muskulatur zusammen, die die Frakturfragmente hervorragend mitperfundiert. Andererseits sind beim Humerus die ästhetischen und funktionellen Ansprüche im Vergleich zu Tibia und Femur geringer. Schulter- und Ellenbogengelenke vermögen aufgrund ihrer guten Mobilität Achsenfehlstellungen bis $\mathrm{zu}$ $20^{\circ}$ auszugleichen, Verkürzungen wirken sich beim Humerus, der nicht primär das Körpergewicht zu tragen hat, wesentlich geringer aus. Neben Desault- und Gilchristverbänden (Abb.3) werden der Oberarmgips mit Schulterkappe, aber auch der Oberarm-Brace nach Sarmiento eingesetzt. Der „Hanging-cast“ mit Distraktion gehört nicht mehr in das klassische Repertoire. Bei Quer- und kurzen Schrägfrakturen wird eine höhere Rate verspäteter Heilungen oder gar Pseudarthrosen beobachtet, da diese Frakturen nur geringe Fragmentkontaktzonen aufweisen und oft sehr instabil sind. Ein enger Kontakt der Hauptfragmente kann bei langen Spiralfrakturen durch interponierte Muskelanteile ebenfalls verhindert werden.

Ungünstig für eine konservative Behandlung erweisen sich inkooperative Patienten, wie Drogen- und Alkoholabhängige, aber auch senile Patienten. Nicht unterschätzt werden darf die Problematik adipöser Patienten mit reduzierter Stabilisierungspotenz des Gipses.

An die Grenzen kommt die konservative Behandlung bei bilateralen Frakturen, dem frei flottierenden Ellenbogen und der Kombination einer Humerusfraktur mit Thoraxtrauma, oder auch die Kombination mit einer Läsion des $\mathrm{N}$. radialis. 


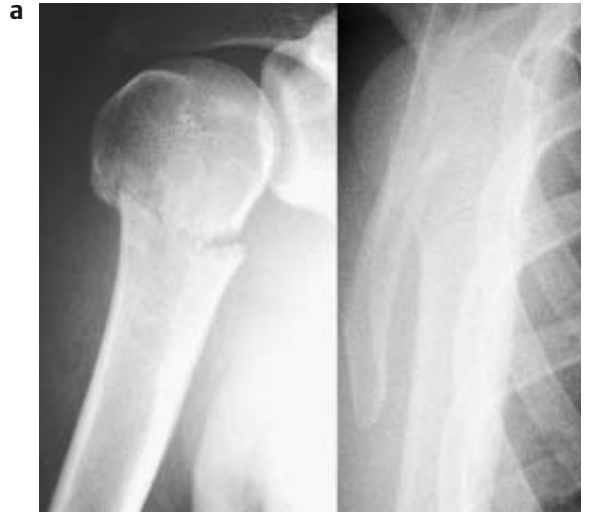

b

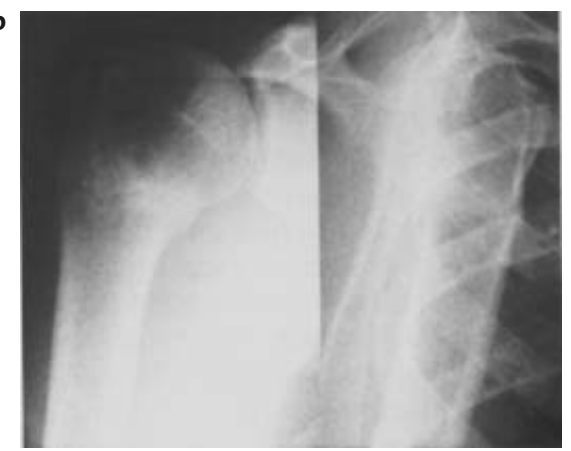

Abb. 1 23-jähriger Patient mit subcapitaler Humerusfraktur rechts nach Sturz auf den ausgestreckten Arm. (a) Unfallbild. (b) Ausheilungsbild unter frühfunktioneller konservativer Therapie.

Sicher nicht für das konservative Vorgehen geeignet sind offene und pathologische Frakturen, zusätzliche Gefäßverletzungen, polytraumatisierte Patienten und Pseudarthrosen. Die rasche Mobilisation nach Humerusmarknagelung führt dazu, dass auch Patienten selbst die operative Therapie zuweilen fordern.

Kindliche Humerusschaftfrakturen allerdings sind weiterhin eine Domäne der konservativen Behandlung.

Die distale Humerusfraktur ist als Folge der Muskelwirkung auf die jeweiligen Fragmente fast ausnahmslos disloziert. Ihre Behandlung ist somit in der Regel operativ. Seltene Ausnahmen können undislozierte, schalenförmige Absprengungen sein, wobei gerade an den Bandansätzen zu beachten ist, dass hier späte Dislokationen zu erwarten sind.

Die Radiusköpfchenfraktur mit einer Stufenbildung von weniger als $2 \mathrm{~mm}$ kann auch heute noch im Oberarmgips mit guten Ergebnissen behandelt werden (Abb.4). Alle darüber hinausführenden
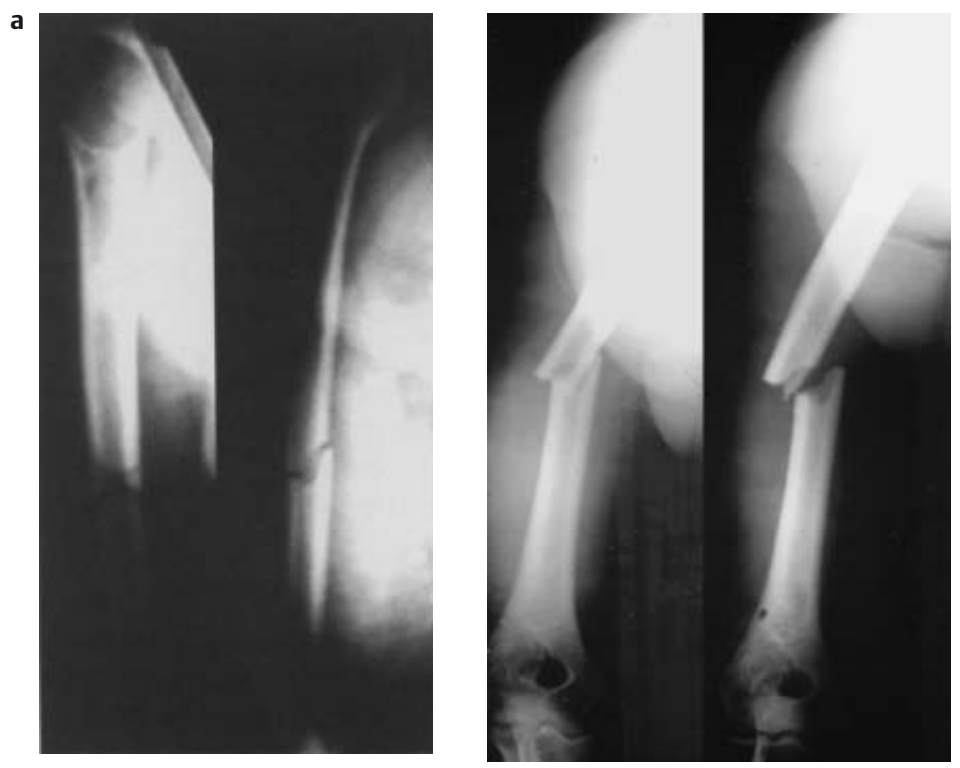

b
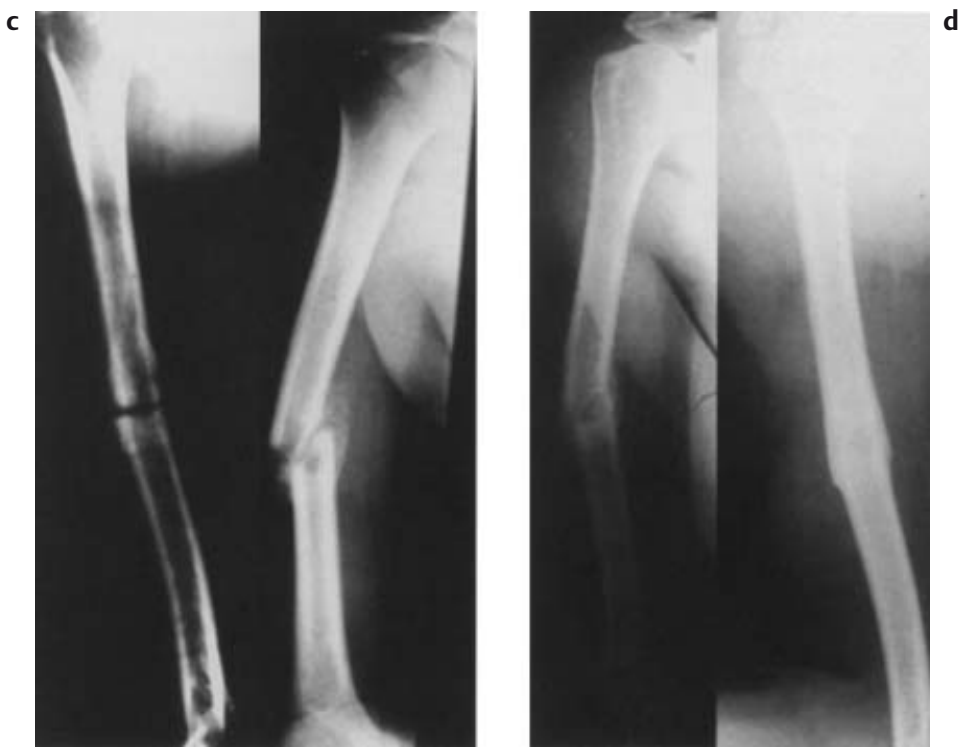

Abb. 2 45-jährige Patientin mit Humerusschaftfraktur 12-A2 rechts nach Sturz auf den Arm. (a) Unfalltag, Retention im Oberarmgips. (b) 2. Tag nach Unfall: Dislokation im Gips. (c) 3 Wochen nach erneuter Reposition im Oberarmgips, bereits angedeutete Kallusbildung. (d) 6 Monate nach Unfall: Knöchern konsolidierte Fraktur unter leichter Achsabweichung, welche funktionell und kosmetisch unbedeutend eingestuft wird.

Verletzungssituationen sollten anatomisch rekonstruiert werden, was im Gips schwer zu reponieren und retinieren ist. Die unverschobene Olekranonfraktur ist aufgrund des starken Muskelzuges konservativ kaum ohne Gefahr der sekundären Dislokation im Gips zu fixieren und scheidet von daher für ein konservatives Verfahren aus.

Erfahrungen in der konservativen Behandlung von Unterarmfrakturen sind beim Erwachsenen als schlecht zu bezeichnen. $\mathrm{Zu}$ hoch sind Pseudarthrose- raten und Achsen- wie auch Rotationsfehlstellungen. Beim Kind wird die konservative Behandlung der Unterarmfraktur noch kontrovers diskutiert. Hingegen stellt die konservative Behandlung verschiedener geschlossener distaler Radiusfraktur loco tipico einen auch heute noch gültigen Standard dar, der allerdings reichlich Erfahrung hinsichtlich Reposition und Fixation erfordert. Als hierfür geeignet sind vor allem extraartikuläre, einfach impaktierte Frakturen des distalen Radius (23-A2) wie auch partielle sagittale artikuläre Frakturen (23-B1), in 

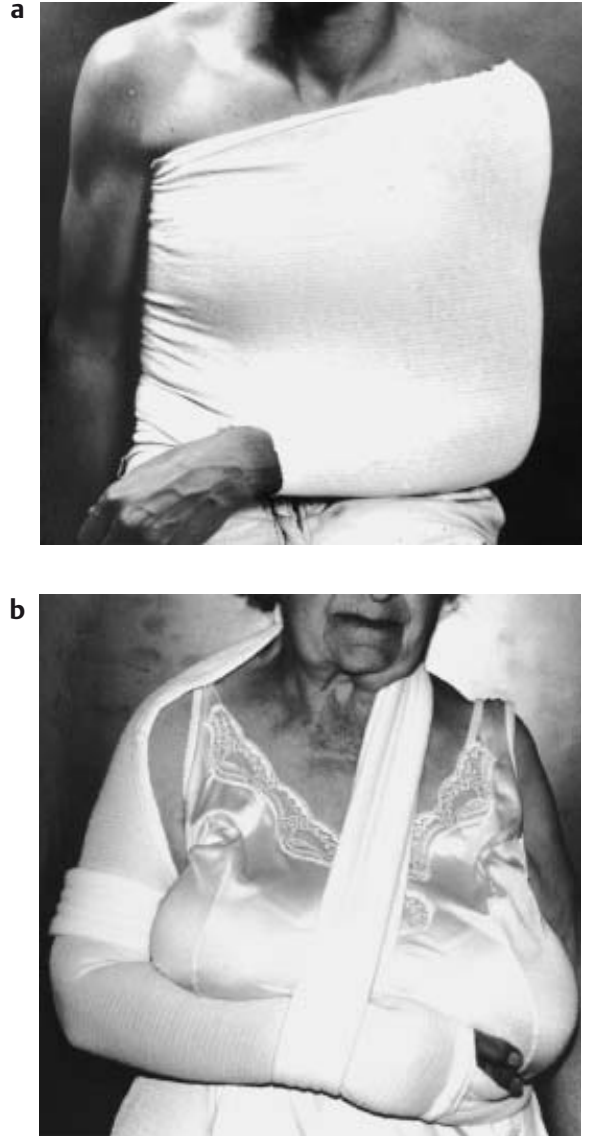

Abb. 3 Desault-Verband (a). Gilchrist-Verband (b).

Abhängigkeit von Alter und Repositionsergebnis auch mehrfragmentäre extraartikuläre Frakturen (23-A3) anzusehen (Abb.5). Bei unverschobenen Frakturen erfolgt direkt die Anlage einer dorsalen oder dorso-volarer Unterarmgipsschiene. Bei dislozierten Frakturen geht diesem die geschlossene Reposition in Bruchspaltanästhesie, vorzugsweise im Aushang, voraus. Alle anderen Frakturtypen sind selten konservativ in akzeptabler Stellung zur Ausheilung zu bringen.

Die Behandlung der häufigsten Handwurzelfraktur, der unverschobenen Kahnbeinfraktur im mittleren oder distalen Drittel, erfolgt konservativ (Abb.6). Das heute gültige Behandlungskonzept sieht einen geschlossenen Unterarmcast mit Einschluss des Daumengrundgelenkes für zwölf Wochen vor. Ein früher verwendeter Oberarmgips für die ersten 6 Wochen wird heute nicht mehr als erforderlich erachtet.

Subkapitale Mittelhandknochenfrakturen (meist MC-V) können nach Reposition im partiellen Faustgips meist sehr gut konservativ behandelt werden. Die
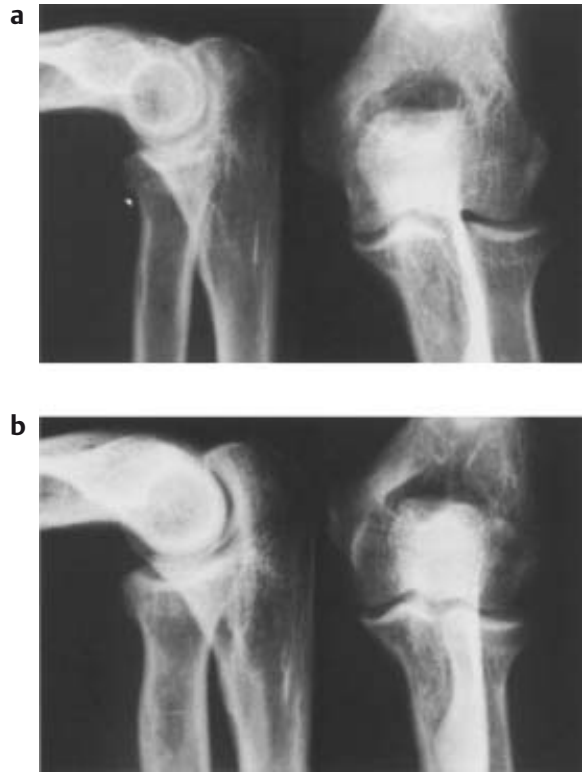

Abb. 4 42-jähriger Patient mit unverschobener Radiusköpfchenfraktur links nach Fahrradsturz. (a) Unfallbild. (b) 5 Wochen nach Unfallereignis ( 3 Wochen Oberarmgips, dann funktionelle Weiterbehandlung).
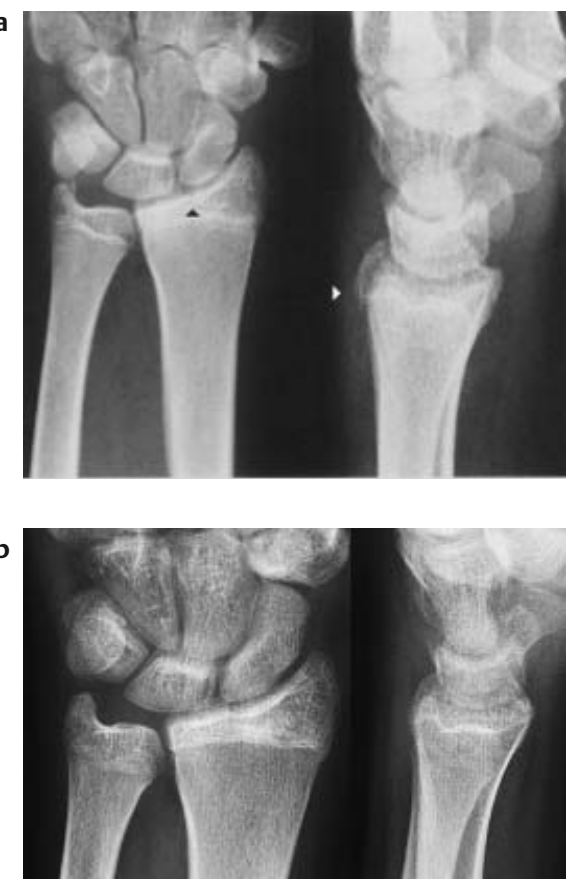

Abb. 5 16-jähriger Patient mit distaler Radiusfraktur rechts mit Gelenkbeteiligung (23-B1) nach Fahrradsturz. (a) Unfallbild. (b) Ausheilungsbild nach 5 Wochen (4 Wochen dorsale Unterarmgipsschiene, 1 Woche funktionelle Weiterbehandlung).

Schaftfraktur an der Mittelhand kann bei guten Längen- und Rotationsverhältnissen ebenfalls konservativ im Gips behandelt werden. Hingegen sind Gelenkfrakturen der MC-I-Basis (Bennett) und der MC-V-Basis (ulnarer Bennett) nicht konservativ in befriedigender Stellung zur Ausheilung zu bringen.

Reine Schaftfrakturen der Mittel- oder Grundfingerglieder können in der Regel durch eine palmare Schiene gut behandelt werden. Hier ist immer eine Streckstellung der Mittelgelenke bei Beugestellung der Grundgelenke anzustreben (Intrinsic-Plus Stellung). Maximal nach 14 Tagen sollte die funktionelle Behandlung folgen.

Kleine Endgelenksfragmente des Endgliedes können in der Stackschen Schiene ebenfalls konservativ behandelt werden, größere Fragmente erfordern hingegen die operative Einpassung und Fixierung.

\section{Konservative Frakturbehandlung an der unteren Extremität}

Der konservativen Frakturbehandlung sind an der unteren Extremität im Vergleich zur oberen wesentlich engere Grenzen gesetzt.

Insbesondere im Bereich des Femurs seien es hüftgelenksnahe Frakturen (Schenkelhals, pertrochantär, subtrochantär), Schaftfrakturen wie auch distale, gelenknahe Frakturen - ist die konservative Behandlung bei Erwachsenen in nahezu allen Fällen insuffizient und obsolet.

Im frühen Wachstumsalter muss der Einsatz von Weichteilextensionen oder Bekken-Beingipsen abgewogen werden.

Die völlig unverschobene Patellalängsfraktur bietet sich der konservativen Therapie mittels eines geschalten Tutors in Streckstellung des Knies und Vollbelastung an. Bei der unverschobenen Querfraktur bleibt zu bedenken, dass auch noch sekundär die Wahrscheinlichkeit einer Distraktion groß ist.

Die konservative Behandlung von nicht verschobenen Frakturen im Bereich der Femurkondylen oder des Tibiakopfes mit einem Oberschenkelgips stellt heute eine seltene Ausnahme dar. Sekundäre Dislokationen im Gips sind bekannt. Handelt es sich um schalenförmige Abbrüche außerhalb der Belastungszonen, kann das konservative Verfahren erwogen werden.

Ansonsten eignen sich Tibiakopffrakturen, insbesondere durch die meist damit vergesellschafteten Impressionen, in der Regel nicht zum konservativen Verfahren, 

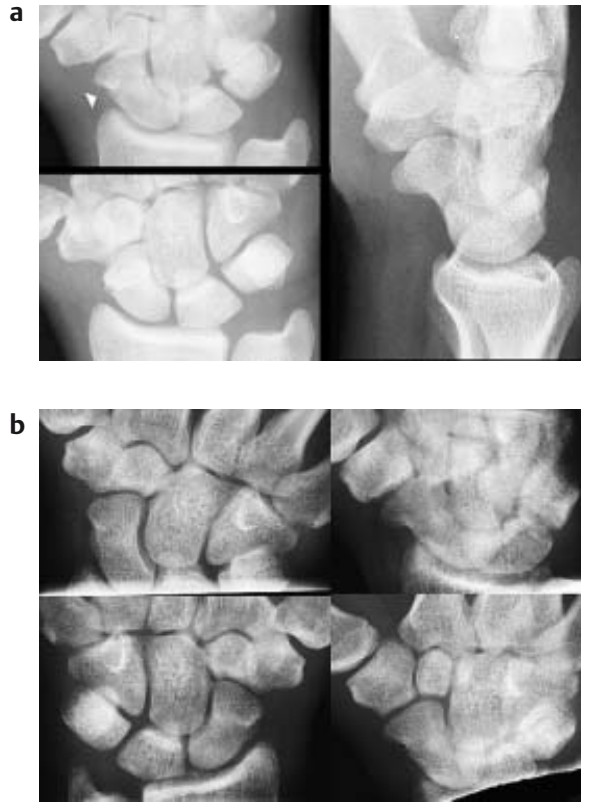

Abb. 6 26-jähriger Patient mit Fraktur des Kahnbeins der rechten Hand. (a) Unfallbild mit wenig verschobener Kahnbeinfraktur. (b) Kahnbeinquartettaufnahmen mit ausgeheilter Fraktur nach 14 Wochen (davon 12 Wochen Oberarmgips mit Daumeneinschluss).

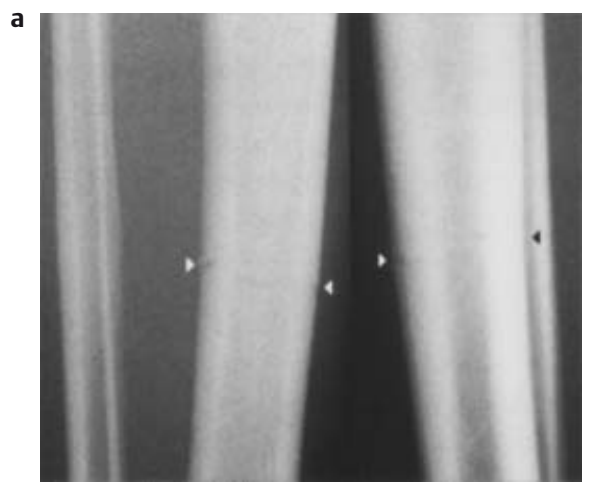

b

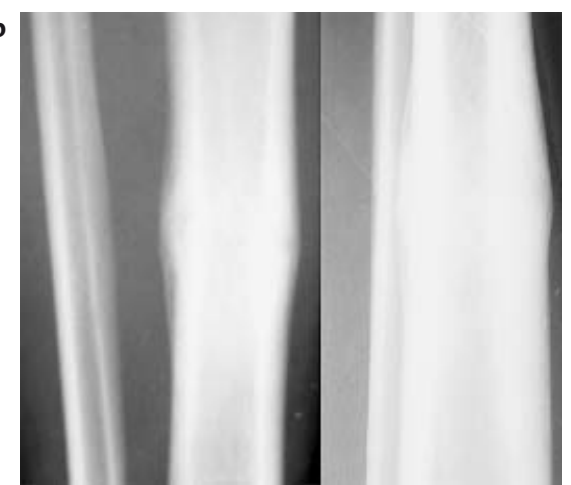

Abb. 7 19-jähriger Patient mit unverschobener Tibiafraktur in Schaftmitte und hoher Fibulafraktur rechts. (a) Details der Unfallaufnahmen in Unterschenkelschaftmitte: komplette, aber unverschobene Tibiafraktur. (b) Details der Aufnahmen bei Ausheilung nach 12 Wochen Gipsbehandlung mit sichtbarer Kallusformation. da eine möglichst anatomische Gelenkrekonstruktion anzustreben ist.

Aufgrund der großen Erfolge der Tibiamarknagelung ist die konservative Behandlung der Tibiaschaftfraktur in den Hintergrund getreten. Dennoch sei auf die vielversprechenden Erfolge Sarmientos mit der Behandlung seines „belowthe-knee casts" hingewiesen. Wenn auch dieser Autor sein konservatives Indikationsspektrum sehr weit stellt, so ist aus unserer Sicht bei Erwachsenen lediglich die unverschobene komplette Unterschenkelfraktur für die Behandlung im Oberschenkelgips, nach Abschwellen im Sarmiento-Brace, geeignet (Abb.7). Die isolierte Tibiaschaftfraktur neigt zur Varusfehlstellung, wodurch sie operativ zu versorgen ist.

Die Fraktur des Pilon-tibiale wird heute nicht konservativ behandelt. Bei Sprunggelenksfrakturen verbietet sich bei Frakturen oberhalb der Syndesmose (Weber C) ebenfalls die konservative Behandlung, um das Risiko der posttraumatischen $\mathrm{Ar}$ throseentstehung zu minimieren.

Undislozierte Frakturen auf Höhe der Syndesmose (Weber B) oder unterhalb der Syndesmose (Weber A) ohne Zusatzverletzungen können unter kritischer Kontrolle konservativ in der Unterschenkelgipsschiene behandelt werden (Abb.8). Allerdings wird man in den meisten Fällen bei kritischer Wertung des Verlaufs doch eine Dislokation feststellen, die dann insbesondere für jüngere $\mathrm{Pa}$ tienten eine OP-Indikation darstellt.

Lediglich unverschobene Frakturen des Talus eignen sich für eine 6-wöchige Gipsbehandlung.

Auch für die Calcaneusfraktur gilt heute, dass dislozierte Frakturen in der Regel operativ angegangen werden sollten, insbesondere bei Gelenkbeteiligung. Entscheidet man sich bei der unverschobenen Calcaneusfraktur für die konservative Behandlung, so darf diese aufgrund der prekären Weichteilsituation nicht im Gips fixiert werden, sondern muss frühfunktionell ohne Belastung erfolgen. Allenfalls ist eine Schiene zur Vermeidung einer Spitzfussstellung zu ergänzen. Je nach Frakturheilung ist hier von einem Entlastungszeitraum von bis $\mathrm{zu} 12$ Wochen auszugehen.

Unter den vielen Frakturmöglichkeiten des Fusses sei nur die häufigste, die Jones-Fraktur der metatarsalen Basis $V$, her- vorgehoben. Lediglich komplett unverschobene Frakturen sollten heute für 6 Wochen im Gips ruhiggestellt werden. Und auch hier sind sekundäre Fragmentdislokationen durch Sehnenzug häufig, wobei diese Diastase ein hohes Pseudarthroserisiko beinhaltet, sodass das operative Verfahren ernsthaft erwogen werden sollte.

\section{Konservative Frakturbehandlung des Schädels und der HWS}

Bei den Schädelfrakturen unterscheidet man topografisch zwischen Kalottenfraktur, Schädelbasisfrakturen und Gesichtsschädelfrakturen. Die meisten Kalottenfrakturen, vor allem Frakturen ohne Dislokation oder in den Frakturspalt verkeiltes Fremdmaterial, und Impressionfrakturen mit geringer Dislokation unter Kalottenbreite werden konservativ behandelt mit strenger Immobilisation, engmaschiger stationärer Überwachung für mehrere Tage und neurologischer, ggf. radiologischer (Schädel-CT) Verlaufskontrolle des Patienten, um posttraumatisch auftretende Hirnblutungen sofort zu erkennen und entsprechend operativ $\mathrm{zu}$ behandeln.

Auch Schädelbasisfrakturen, obwohl wegen ihrer Nähe zu den austretenden Hirnnerven und des Hirnstammes als komplikationsreich betrachtet, werden in gleicher Weise behandelt. Bei offenen Frakturen und Liquoraustritt, sowie bei Gehirnverletzungen ist hierbei allerdings eine operativer Eingriff indiziert. Anders liegt die Situation bei Gesichtsschädelfrakturen. Da hier doch wesentliche Funktionenstörungen, z.B. der Kaubewegung von Ober und Unterkiefer, oder Sehstörungen bei Orbitafrakturen auftreten können ist hier eine operative Versorgung angezeigt. Lediglich bei nicht verschobenen Frakturen ohne funktionelle Störungen wird eine konservative Therapie z.B. mit Ruhigstellung des Unterkiefers durchgeführt.

Bei der Entscheidung über die Behandlung von HWS-Frakturen spielt die Beurteilung der Stabilität, die sich nach dem Ausmaß der Schädigung ossärer und disco-ligamentärer Strukturen eines oder mehrerer Segmente entscheidet, eine zentrale Rolle. Gleichzeitig muss eine mögliche Schädigung des Rückenmarks in Betracht gezogen werden, bei der die operative Dekompression des Spinalkanals erfolgt. 

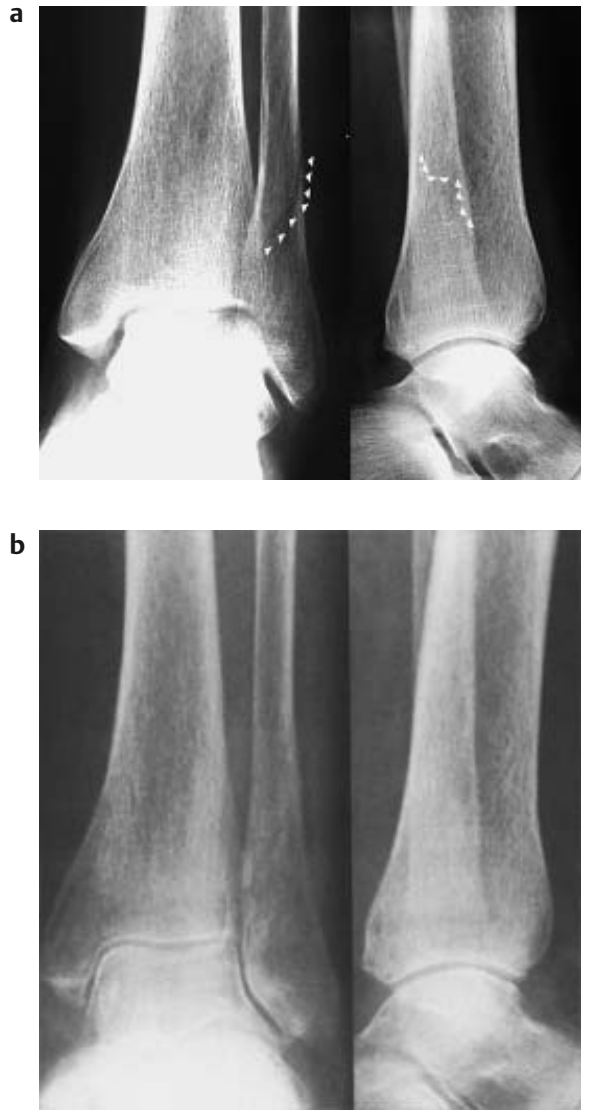

Abb. 8 66-jährige Patientin mit unverschobener Sprunggelenksfraktur auf Syndesmosenhöhe links. (a) Unfallaufnahmen. (b) Konsolidierte Fraktur, Kontrolle nach 3 Monaten (Unterschenkelgips für 6 Wochen).

Bei Halswirbelfrakturen werden im Bereich der oberen HWS Frakturen der occipitalen Kondylen ohne Instabilität durch eine SOMI-Stütze $($ SOMI = Sternal occiput mandibular immobilization) für 6 Wochen behandelt. Ein starrer Metallrahmen, der sich auf dem Sternum abstützt und mit einer Kinn und Hinterhauptstütze verbunden ist, schient die HWS von außen und schränkt die Beweglichkeit der HWS deutlich ein. Nachteil ist ihr geringer Komfort und die Gefahr von Druckstellen.

Durch einen Halo-Fixateur (Abb.9) werden Atlasfrakturen, atlantoaxiale Instabilitäten, Densfrakturen (Typ I und III) sowie C2-Bogenfrakturen (hangman-Fractures), die gering oder nicht disloziert sind, behandelt. Der Halo-Fixateur besteht aus einem Halo-Ring, der mit 4 Schrauben in der Schädelkalotte befestigt wird. Über ein Gestell ist der Halo-Ring stabil mit einer Brust-Schulter-RückenStütze verbunden. Ob dies noch als kon-

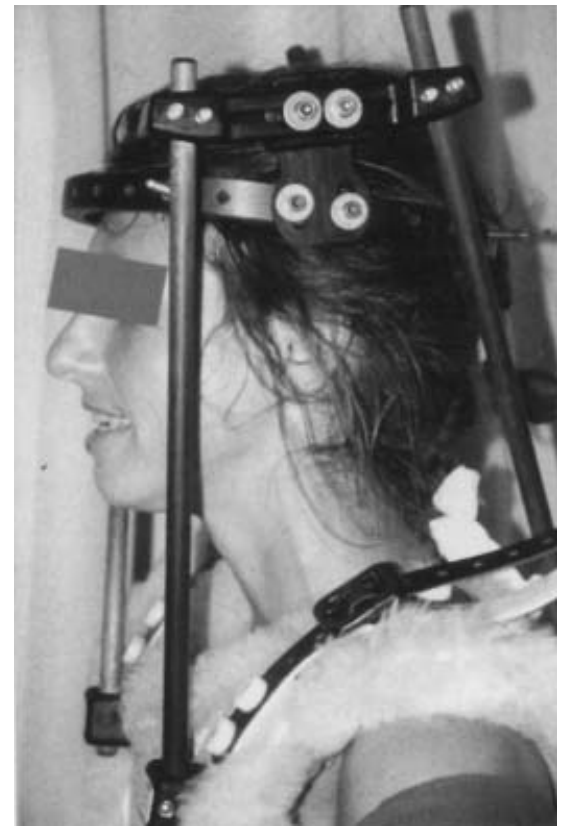

Abb.9 Halo-Fixateur zur konservativen Behandlung von Atlasfrakturen, atlanto-axiale Instabilitäten, Densfrakturen (Typ I und III) sowie C2-Bogenfrakturen (hangman-fractures), die gering oder nicht disloziert sind.

servatives oder in seiner Nähe zum Fixateur externe als operatives Verfahren anzusehen ist, bleibt diskutabel. Alle anderen HWS-Frakturen, insbesondere die Dens-Basisfraktur, die bei konservativer Behandlung eine hohe Pseudarthrosenbildung aufweist, können nicht konservativ behandelt werden.

Im Bereich der unteren HWS lassen sich Abriss- und Laminafrakturen ohne Instabilität, sowie Dornfortsatz- und Gelenkfortsatzfrakturen, gut konservativ mit Anlage eines weichen oder harten Kragens für sechs Wochen behandeln. Kompressionsfrakturen ohne Hinterkantenbeteiligung erfordern die Anlage einer SOMI-Stütze.

\section{Konservative Frakturbehandlung von Thorax, BWS, LWS und Becken}

Einfache Rippenfrakturen wie auch Rippenserienfrakturen werden konservativ funktionell therapiert. Dabei sind mögliche Begleitverletzungen durch frakturierte Rippenanteile (Pneumothorax, Nieren, Leber oder Milzverletzungen) auszuschließen. Wichtig sind eine ausreichende medikamentöse Analgesie und das Durchführen einer Atemtherapie zur Vermeidung von Lungenminderbelüftung und Entstehung einer Pneumonie. Beim instabilen Thorax mit paradoxer Atmung kann eine operative Stabilisierung erwogen werden.

Sternumfrakturen werden in der Regel ebenfalls konservativ behandelt. Hier ist auf ausreichende Analgesie und Atemgymnastik, sowie auf Zeichen einer Herzkontusion und Myokardschädigung $\mathrm{zu}$ achten.

Bei Frakturen im BWS- und LWS-Bereich stellt die Beurteilung der intakten Hinterkante der Wirbelkörper einen entscheidenden Faktor für die Stabilität und Intaktheit des Spinalkanales und damit für Indikation zur konservativen Behandlung der Fraktur dar. Weitere Faktoren sind das Ausmaß der Kyphose und das Ausmaß der Spinalstenose. Stabile Frakturen ohne neurologische Ausfälle eignen sich zur konservativen Behandlung. Ziel ist, die Patienten früh unter Beachtung rückenschonender Verhaltensweise (Drehen en bloc, Vermeiden der Beugung und Rotation) unter krankengymnastischer Anleitung zu mobilisieren. Durch systematische Kräftigungsübung der Rumpfmuskulatur wird der Aufbau eines „Dynamischen Muskelmieders“ erreicht. Auch bei Wirbelfrakturen im hohen Alter erfolgt, wenn irgend möglich, die frühe Mobilisation. Immobilisation sollte nur bei heftigen Beschwerden und bei einer frakturierten Hinterkante des Wirbelkörpers erfolgen. Die Verwendung der Lordoselagerung oder eines Lordosegips muss im Einzelfall abgewogen werden. Eine zu lange Immobilisation ist aber mit deutlichen Nachteilen wie erhöhtem Thromboserisiko, Pneumonie und allgemeinem Muskelabbau verbunden. Aus diesen Gründen ist bei frakturierter Hinterkante auch ohne neurologische Ausfälle eine operative Versorgung angezeigt.

Die überwiegende Anzahl der Beckenfrakturen wird auch heute weiterhin konservativ behandelt. Dies betrifft in erster Linie alle stabilen und wenig dislozierten Frakturen (Typ-A-Verletzungen und ausgewählte Fälle von Typ-B-Verletzungen). Die Mobilisation kann prinzipiell schon am 1.Tag nach Unfall erfolgen und wird lediglich durch das Ausmaß der Schmerzen limitiert. Auch die betroffene Seite kann vollständig belastet werden, bei stärkeren Schmerzen mit kurzzeitiger Entlastung an Unterarmgehstützen.

Die Indikation zur konservativen Behandlung besteht bei unverschobenen Acetabulumfrakturen und Frakturen außerhalb 
des gewichtstragenden Teils. Immer muss jedoch die Gelenkkongruenz gegeben sein. Ebenso wie bei den anderen stabilen Beckenfrakturen wird hierbei eine frühfunktionelle Therapie unterhalb der Schmerzgrenze angestrebt, das gestreckte Anheben des Beines sollte vermieden werden. Die Mobilisation sollte bei strikter Entlastung der betroffenen Extremität an Unterarmgehstützen, je nach Frakturtyp für mindestens 6 Wochen, erfolgen.

\section{Komplikationen der konservativen Frakturbehandlung}

Wie operative Verfahren, so trägt auch die konservative Behandlung Komplikationsrisiken in sich.

Aufgrund des Risikos der Zirkulationsstörung dürfen frische Frakturen wegen der ihnen eigenen Schwellneigung im Frakturbereich nicht zirkulär eingegipst werden. Die Verwendung von Gipsschienen oder das direkte und komplette Spalten eines frischen, aber bereits trockenen zirkulären Gipses ist notwendig.

Aber auch dann noch gilt es, venöse oder arterielle Abflussstörungen auszuschließen bzw. zu behandeln.

Gefürchtete Komplikationen in diesem Zusammenhang sind die Extremitätengangrän, wie auch ischämische Muskelkontrakturen bzw. das KompartmentSyndrom, insbesondere an Unterschenkel und Ellenbogen.

Drucknekrosen der Haut oder gar darunterliegender Sehnen gelten als Komplikationen der Gipsbehandlung.

Äußerungen über Schmerzen unter dem Gips dürfen niemals heruntergespielt werden und erfordern die sorgfältige Prüfung.

Aufgrund erhöhten Druckes können auch Druckschäden oberflächlicher Nerven entstehen. Insbesondere im Bereich des $\mathrm{N}$. peroneus am Wadenbeinköpfchen, wie auch des N. ulnaris an der Innenseite des Ellenbogengelenkes muss die Polsterung ausreichend sein.
Das erhöhte Risiko der Entwicklung von Thrombosen oder gar Embolien aufgrund der Gipsretention ist bekannt.

Die antithrombotische Behandlung mit niedermolekularen Heparinen wie auch beispielsweise das Training der Wadenmuskulatur im Unterschenkelgips zur Verbesserung der Rheologie sind dringend zu empfehlen. Auf frühe Anzeichen einer Thrombose ist rasch und konsequent zu reagieren.

Das Ausbleiben der knöchernen Heilung unter Gipsbehandlung ist als Komplikation zu betrachten. Bildet sich trotz mangelnder Heilung ein eindrucksvoller Kallus an den jeweiligen Frakturenden, so spricht man von hypertrophen Pseudarthrosen. Trotz osteogenetischer Potenz besteht hier ein Mangel an Stabilität. In der Regel folgt der Wechsel zur operativen Stabilisierung. Findet sich auch diese Kallusformation nicht, so handelt es sich um eine hypotrophe Pseudarthrose, die neben der ausreichenden Stabilisierung in der Regel auch eine zusätzliche Spongiosaplastik benötigt.

Fazit

Aus der Tatsache, dass sowohl die konservativen als auch die operativen Verfahren über ein ihnen eigenes Spektrum an Vorteilen wie auch Nachteilen und Komplikationen verfügen, macht deutlich, dass auch heute keine pauschale Bevorzugung einer der beiden Alternativen möglich ist. Es gilt die Überlegenheit des einzelnen Verfahrens für die jeweils spezifische Situation herauszuarbeiten. Dieser Prozess fußt auf unfallchirurgischer Erfahrung, kritischem Abwägen in Anpassung an die jeweilige Fraktur- und Weichteilsituation, aber auch an die Gesamtsituation des Patienten einschließlich dessen Persönlichkeit.

\section{Literatur}

${ }^{1}$ Böhler L. Die Technik der Knochenbruchbehandlung. Maudrich, Wien 1951

2 Böhler L. Gegen die operative Behandlung von frischen Oberarmschaftbrüchen. Langenbecks Arch Klin Chir 1964; 308: 465475

${ }^{3}$ Hertel P. Tibiakopffrakturen. Unfallchirurg 1997; 100: 508-523

${ }^{4}$ Kayser M, Muhr G, op den Winkel R, Ekkernkamp A. Funktionelle Behandlung der Humerusschaftfraktur nach Sarmiento: Ergebnisse nach 3-jähriger Erfahrung. Unfallchirurg 1986; 89: 253-258

${ }^{5}$ Moschinski D. Der traumatologische Notfall - Frakturen. In: Kremer, K., E. Müller (eds.): Die chirurgische Poliklinik. Thieme, Stuttgart, New York 1987

${ }^{6}$ Nordqvist A, Petersson CJ, Redlund-Johnell I. Mid-clavicle fractures in adults: end result study after conservative treatment. J Orthop Trauma 1998: 12: 572-576

${ }^{7}$ Oestern HJ. Distale Radiusfrakturen. Teil I. Grundlagen und konservative Therapie. Chirurg 1999; 70: 1180-1192

${ }^{8}$ Richter J, Schulze W, Muhr G. Stabile Knöchelbrüche - Indikation zur Operation oder konservativen Therapie? Orthopäde 1999; 28: $493-499$

${ }^{9}$ Rodriguez-Merchan EC. Management of comminuted fractures of the distal radius in the adult. Conservative or surgical? Clin Orthop 1998; 353: 53-62

${ }^{10}$ Rudigier J. Kurzgefasste Handchirurgie. Hippokrates, Stuttgart 1997

${ }^{11}$ Sarmiento A. A functional below-the-knee cast for tibial fractures. J Bone Jt Surg 1967; 49 A: 855-859

${ }^{12}$ Szyszkowitz R, Schippinger G. Die Frakturen des proximalen Humerus. Unfallchirurg 1999; 102: 422-428

${ }^{13}$ Thermann H, Hufner T, Schratt HE, et al. Therapie intraartikulärer Fersenbeinfrakturen bei Erwachsenen - Ein Behandlungsalgorithmus. Unfallchirurg 1999; 102: 152 - 166

${ }^{14}$ Von Laer L. Frakturen und Luxationen im Wachstumsalter. Thieme, Stuttgart, New York 1996

PD Dr. med. J. Blum

Oberarzt

Dr. med. A. Noltze

Assistenzarzt

Univ.-Prof. Dr. med. P. M. Rommens

Direktor der Klinik

Klinik und Poliklinik für Unfallchirurgie Johannes Gutenberg-Universität Mainz Langenbeckstr. 1

55131 Mainz 